\title{
THE SUMMABILITY OF THE TRIPLE FOURIER SERIES AT POINTS OF DISCONTINUITY OF THE FUNCTION DEVELOPED*
}

BY

\section{BESS M. EVERSULL}

In a previous papert the author has studied the summability of the development of a function of three variables in a triple Fourier series at points of continuity of the function developed, using the method originated by Fejér and applied by him to problems involving simple series. For the latter series the proof of the summability at points of discontinuity of the first kind (finite jumps) is quite similar to that for points of continuity, and the two cases can be treated by means of a single discussion. In passing to the case of the double Fourier series, it is found that the study of the behavior of the series at points of discontinuity of an analogous type presents difficulties and complications that do not arise in connection with points of continuity. + When we go on to the case of triple series, we find that the difficulties and complications of the corresponding problem are still further increased.

In the present paper a study is made of the summability of the triple Fourier series at points of discontinuity of the type that would be apt to arise in physical applications. This includes discontinuities lying on plane or curved surfaces, and such that the function to be developed approaches the same value as we approach the point along any path lying entirely within the region of continuity.

The definition of summability used in the discussion of the triple series is analogous to that of Cesàro for the simple series. Designating by $s_{l m n}$ the sum of the $l m n$ terms of the triple series $\sum a_{l m n}$ lying in a rectangular parallelepiped $l$ terms high, $m$ terms broad, and $n$ terms deep, in the upper, left-hand, forward corner of this series, and forming

(1) $S_{l m n}^{(r)}=\sum_{i=1, j=1, k=1}^{l, m, n} \frac{\Gamma(r+l-i)}{\Gamma(r) \Gamma(l-i+1)} \cdot \frac{\Gamma(r+m-j)}{\Gamma(r) \Gamma(m-j+1)} \cdot \frac{\Gamma(r+n-k)}{\Gamma(r) \Gamma(n-k+1)} s_{i j k}$,

$$
A_{l m n}^{(r)}=\frac{\Gamma(l+r)}{\Gamma(r+1) \Gamma(l)} \cdot \frac{\Gamma(m+r)}{\Gamma(r+1) \Gamma(m)} \cdot \frac{\Gamma(n+r)}{\Gamma(r+1) \Gamma(n)},
$$

* Presented to the Society, December 28, 1923.

† Annals of Mathematics, ser. 2, vol. 24 (1922), pp. 141-166.

$\ddagger$ A discussion of this case has been given by Professor C. N. Moore (cf. Mathematische Annalen, vol. 74 (1913), pp. 557-572. 
we say that the triple series is summable $(C r)$ to the limit

$$
\lim _{l, m, n \rightarrow \infty} \frac{S_{l m n}^{(r)}}{l m n}
$$

and has the value of that limit, providing such a limit exists.

\section{Discontinuities along Plane surfaces}

The triple Fourier series corresponding to the function $f(x, y, z)$ may by written in the form

(4) $\sum_{l=1, m=1, n=1}^{\infty, \infty, \infty} \frac{1}{2^{E(1 / l)+E(1 / m)+E(1 / n)} \pi^{3}}$

$$
\cdot \int_{-\pi}^{\pi} \int_{-\pi}^{\pi} \int_{-\pi}^{\pi} f\left(x^{\prime}, y^{\prime}, z^{\prime}\right) P_{l m n}\left(x, y, z, x^{\prime}, y^{\prime}, z^{\prime}\right) d x^{\prime} d y^{\prime} d z^{\prime},
$$

where

$$
\begin{aligned}
& P_{l m n}\left(x, y, z, x^{\prime}, y^{\prime}, z^{\prime}\right) \\
& \quad=\cos \left[(l-1)\left(x^{\prime}-x\right)\right] \cos \left[(m-1)\left(y^{\prime}-y\right)\right] \cos \left[(n-1)\left(z^{\prime}-z\right)\right]
\end{aligned}
$$

and $E(u)$ represents the largest integer contained in $u$.

We wish to investigate first the behavior of the series at a point of discontinuity such that all other points of discontinuity in its neighborhood lie on a plane passing through that point and such that $f(x, y, z)$ approaches a definite value as we approach the point of discontinuity from either side of the plane. Several lemmas are necessary before we can prove the fundamental theorem. The first two are merely stated, their proofs appearing in a previous paper by the writer.*

Lemma 1. Let $R$ be a region in space lying within the cube whose sides are $\alpha= \pm\left(\pi-\varrho_{1}\right), \beta= \pm\left(\pi-\varrho_{1}\right), \gamma= \pm\left(\pi-\varrho_{1}\right)$, and outside the sphere of radius $\varrho_{2}$ whose center is at the origin, where $\varrho_{1}$ and $\varrho_{2}$ are two positive constants whose sum is less than $\pi$. Then if $\varphi(\alpha, \beta, \gamma)$ is finite and integrablet in $R$, the limit

$$
\lim _{l, m, n \rightarrow \infty} \frac{1}{\ln n} \iiint_{R} \varphi(\alpha, \beta, \gamma) \frac{\sin ^{2} l \alpha}{\sin ^{2} \alpha} \frac{\sin ^{2} m \beta}{\sin ^{2} \beta} \frac{\sin ^{2} n \gamma}{\sin ^{2} \gamma} d \alpha d \beta d \gamma
$$

will exist and be equal to zero.

* Loc. cit., pp. 154-156, 157-158.

† Here and elsewhere thoughout the paper, when it is said that a function is integrable, it is meant that it has an integral according to the definition of Lebesgue. 
Lemma 2. Let $R$ be a region within the cube described in Lemma 1, and such that the point $\alpha=\beta=\gamma=0$ lies uithin or on the boundary of $R$. Then if $\varphi(\alpha, \beta, \gamma)$ is finite and integrable in $R$, and if

$$
\lim _{\alpha, \beta, \gamma \rightarrow 0} \varphi(\alpha, \beta, \gamma)=0
$$

the limit

$$
\lim _{l, m, n \rightarrow \infty} \frac{1}{l m n} \iiint_{R} \varphi(\alpha, \beta, \gamma) \frac{\sin ^{2} l \alpha}{\sin ^{2} \frac{\sin ^{2} m \beta}{\alpha}} \frac{\sin ^{2} n \gamma}{\sin ^{2} \beta} d \alpha d \beta d \gamma
$$

will exist and be equal to zero.

Lemma 3. If $g, h$ and $k$ are positive numbers less than $\pi$, the limit

$$
\lim _{l, m, n \rightarrow \infty} \frac{1}{\operatorname{lm} n} \int_{0}^{g} \int_{0}^{h} \int_{0}^{k} \frac{\sin ^{2} l \alpha}{\operatorname{lin}^{2} \alpha} \frac{\sin ^{2} m \beta}{\sin ^{2} \beta} \frac{\sin ^{2} n \gamma}{\sin ^{2} \gamma} d \alpha d \beta d \gamma
$$

and the seven others in which the limits of integration are respectively $-g$ to 0,0 to $h, 0$ to $k ; 0$ to $g,-h$ to 0,0 to $k ; 0$ to $g, 0$ to $h$, $-k$ to $0 ;-g$ to $0,-h$ to 0,0 to $k ;-g$ to 0,0 to $h,-k$ to 0 ; 0 to $g,-h$ to $0,-k$ to $0 ;-g$ to $0,-h$ to $0,-k$ to 0 , will each exist and be equal to $\frac{1}{8}$.

The proof for the first limit follows easily from Lemma 1 and a result due to Fejér.* The succeeding seven cases may be reduced to the first by a suitable change of variable. An analogous proof is given under Lemma 5 of the writer's previous paper.

Lемма 4. If $g, h$ and $k$ are positive numbers less than $\pi$ and we divide the parallelepiped whose sides are $\alpha= \pm g, \beta= \pm h, \gamma= \pm k$ into two parts $R_{1}$ and $R_{2}$ by passing a plane through the diagonal joining two opposite vertices, the limits

$$
\begin{array}{r}
\lim _{l, m, n \rightarrow \infty}\left\{\frac{1}{l m n \pi^{3}} \iiint_{R_{1}} \frac{\sin ^{2} l \alpha}{\sin ^{2} \alpha} \frac{\sin ^{2} m \beta}{\sin ^{2} \beta} \frac{\sin ^{2} n \gamma}{\sin ^{y} \gamma} d \alpha d \beta d \gamma\right\}, \\
\lim _{l, m, n \rightarrow \infty}\left\{\frac{1}{l m n \pi^{3}} \iiint_{R_{3}} \frac{\sin ^{2} l \alpha}{\sin ^{2} \alpha} \frac{\sin ^{2} m \beta}{\sin ^{2} \beta} \frac{\sin ^{2} n \gamma}{\sin ^{2} \gamma} d \alpha d \beta d \gamma\right\}
\end{array}
$$

will exist and will each equal $\frac{1}{2}$.

Suppose the plane passes through the diagonal joining the vertices $(g, h, k)$ and $(-g,-h,-k)$. In general this plane will cut through two pairs of faces. Suppose for the sake of definiteness that we assume it cuts the

*Mathematische Annalen, 58 (1904), p. 55. 
faces $\alpha= \pm g, \beta= \pm h$. We shall chose $R_{1}$ as the region included between the dividing plane and the planes $\alpha= \pm g, \beta= \pm h, \gamma=-k$, and show that the first limit in (6) exists and is equal to $\frac{1}{2}$. It will then follow that the second of these limits exists and is equal to $\frac{1}{2}$, as we know that the sum of the two expressions in brackets approaches the limit unity as $l, m$ and $n$ become infinite.

The equation of the plane may be written in the form $\gamma=p \alpha+q \beta$ where $p$ and $q$ are constants. The quantity in brackets in the first of expressions (6) may then be written

$$
\begin{aligned}
\frac{1}{\operatorname{lm} n \pi^{3}}\left\{\int_{0}^{g} \int_{0}^{h} \int_{-k}^{0}\right. & +\int_{0}^{g} \int_{0}^{h} \int_{0}^{p \alpha+q \beta}+\int_{0}^{g} \int_{-h}^{0} \int_{-k}^{p \alpha+q \beta} \\
& \left.+\int_{-g}^{0} \int_{-h}^{0} \int_{-k}^{p \alpha+q \beta}+\int_{-g}^{0} \int_{0}^{h} \int_{-k}^{p \alpha+q \beta}\right\},
\end{aligned}
$$

where the quantity under the integral sign in each case is the same as that under the integral sign in the expression (6).

The first term of (7) approaches $\frac{1}{8}$ as a limit as $l, m$ and $n$ become infinite, by Lemma 3. If we make the substitution $\alpha=-\alpha^{\prime}, \beta=-\beta^{\prime}$, $\gamma=-\gamma^{\prime}$ in the third and fourth terms, the third combines with the fifth to form an expression which by Lemma 3 approaches $\frac{1}{4}$ as a limit as $l, m$ and $n$ become infinite, and the fourth combines with the second to form an expression which approaches $\frac{1}{8}$ as $l, m$ and $n$ become infinite, by the same lemma. Hence in this case the limit of the first of expressions (6) exists and is equal to $\frac{1}{2}$.

The plane through the diagonal joining the vertices $(g, h, k)$ and $(-g,-h,-k)$ may also be perpendicular to one of the planes, say to $\gamma=k$. In this case we take $R_{1}$ to be the region included between the plane through the diagonal and the planes $\alpha=g, \beta=-h, \gamma= \pm k$. The quantity in brackets in the first of expressions (6) will then be

$$
\frac{1}{\operatorname{lmn} \pi^{3}}\left\{\int_{0}^{g} \int_{-h}^{0} \int_{-k}^{k}+\int_{0}^{g} \int_{0}^{h \alpha / g} \int_{-k}^{k}+\int_{-g}^{0} \int_{-h}^{h \alpha / g} \int_{-k}^{k}\right\}
$$

where the expression under the integral signs is the same as in (6). From Lemma 3 the first term of this expression approaches $\frac{1}{4}$ as $l, m$ and $n$ become infinite. If in the third term we make the change of variable

$$
\alpha=\alpha^{\prime}, \quad \beta=\beta^{\prime},
$$


this term combines with the second to produce an expression which, from Lemma 3, also approaches $\frac{1}{4}$ as $l, m$ and $n$ become infinite, and hence for this case also the first limit in (6) exists and is equal to $\frac{1}{2}$.

Hence this is true for all positions of the plane through the diagonal, and as shown before, the second limit in (6) must also exist and be equal to $\frac{1}{2}$ for all positions of the plane. The lemma is therefore proved.

Lemma 5. If $R$ is the region described in Lemma 2, the limit

$$
\begin{aligned}
\lim _{l, m, n \rightarrow \infty}\left[\frac{1}{l m n} \iiint_{R} \frac{\sin ^{2} l \alpha}{\sin ^{2} \alpha} \frac{\sin ^{2} m \beta}{\sin ^{2} \beta} \frac{\sin ^{2} n \gamma}{\sin ^{2} \gamma} d \alpha d \beta d \gamma\right. \\
\\
\left.\quad-\frac{1}{l m n} \iiint_{R} \frac{\sin ^{2} l \alpha}{\alpha^{2}} \frac{\sin ^{2} m \beta}{\beta^{2}} \frac{\sin ^{2} n \gamma}{\gamma^{2}} d \alpha d \beta d \gamma\right]
\end{aligned}
$$

will exist and be equal to zero.

The expression in brackets may be written in the form

$$
\begin{aligned}
\frac{1}{\operatorname{lm} n} \iiint_{R}\left(1-\frac{\sin ^{2} \alpha}{\alpha^{2}}\right)\left(1-\frac{\sin ^{2} \beta}{\beta^{2}}\right) & \left(1-\frac{\sin ^{2} \gamma}{\gamma^{2}}\right) \\
& \cdot \frac{\sin ^{2} l \alpha}{\sin ^{2} \alpha} \frac{\sin ^{2} m \beta}{\sin ^{2} \beta} \frac{\sin ^{2} n \gamma}{\sin ^{2} \gamma} d \alpha d \beta d \gamma .
\end{aligned}
$$

It may easily be seen that the product of the three terms in parentheses under the integral sign satisfies the condition imposed on $\varphi(\alpha, \beta, \gamma)$ in Lemma 2 , and hence the lemma follows at once.

Lemma 6. Let $R$ be a region within the cube described in Lemma 1, and without the cube $-\varepsilon \leqq \alpha \leqq \varepsilon,-\varepsilon \leqq \beta \leqq \varepsilon,-\varepsilon \leqq \gamma \leqq \varepsilon$, where $\varepsilon$ is an arbitrarily small positive quantity and $\varphi(\alpha, \beta, \gamma)$ a function that is integrable in $R$ and remains finite in that part of $R$ which includes all points whose coördinates satisfy one or two of the conditions

$$
-\varepsilon \leqq \alpha \leqq \varepsilon, \quad-\varepsilon \leqq \beta \leqq \varepsilon, \quad-\varepsilon \leqq \gamma \leqq \varepsilon
$$

Then the limit

(9) $\lim _{l, m, n \rightarrow \infty} \frac{1}{l m n} \iiint_{R} \varphi(\alpha, \beta, \gamma) \frac{\sin ^{2} l \alpha}{\sin ^{2} \alpha} \frac{\sin ^{2} m \beta}{\sin ^{2} \beta} \frac{\sin ^{2} n \gamma}{\sin ^{2} \gamma} d \alpha d \beta d \gamma$

will exist and be equal to zero.

The quantity in (9) may be written in the form

$$
\frac{1}{\operatorname{lm} n}\left\{\iiint_{R^{\prime}}+\iiint_{R^{\prime}}\right\}
$$


where the integrands are the same as that in (9), $R^{\prime}$ that part of $R$ which includes all points whose coordinates satisfy one or two of the conditions (8), and $R^{\prime \prime}$ the remainder of $R$.

By Lemma 1 the first term of (10) approaches zero as a limit as $l, m$ and $n$ become infinite.

Consider now the second term of (10):

$$
\begin{array}{r}
\left|\frac{1}{l m n} \iiint_{R^{\prime}} \varphi(\alpha, \beta, \gamma) \frac{\sin ^{2} l \alpha}{\sin ^{2} \alpha} \frac{\sin ^{2} m \beta}{\sin ^{2} \beta} \frac{\sin ^{2} n \gamma}{\sin ^{2} \gamma} d \alpha d \beta d \gamma\right| \\
\quad<\frac{1}{l m n \sin ^{6} \eta} \iiint_{R^{\prime}}|\varphi(\alpha, \beta, \gamma)| d \alpha d \beta d \gamma
\end{array}
$$

where $\eta$ is the smaller of the quantities $\varepsilon$ and $\delta, \delta$ being the least distance between $R^{\prime}$ and the cube $\alpha= \pm \pi, \beta= \pm \pi, \gamma= \pm \pi$. The right-hand side of this inequality may be made as small as we please by choosing $l, m$ and $n$ sufficiently large and therefore the second term of (10) approaches zero as $l, m$ and $n$ become infinite. Hence the limit (9) exists and is equal to zero and the lemma is proved.

Before proving the next theorem we shall define an expression which we wish to use in the statement of this and succeeding theorems. A critical region associated with the point $\left(x_{1}, y_{1}, z_{1}\right)$ is defined as a region made up of points whose coordinates satisfy one or more of the inequalities

$$
x_{1}-\varepsilon \leqq x \leqq x_{1}+\varepsilon, \quad y_{1}-\varepsilon \leqq y \leqq y_{1}+\varepsilon, \quad z_{1}-\varepsilon \leqq z \leqq z_{1}+\varepsilon,
$$

where $\varepsilon$ is an arbitrarily small positive quantity.

We are now ready to prove the following theorem:

THEOREM I. If $f(x, y, z)$ is integrable in the region

$$
(-\pi \leqq x \cdot \pi, \quad-\pi \leqq y \leqq \pi, \quad-\pi \leqq z \leqq \pi)
$$

and $\left(x_{1}, y_{1}, z_{1}\right)$ is a point of discontinuity of $f(x, y, z)$ such that every other point of discontinuity in the neighborhood of $\left(x_{1}, y_{1}, z_{1}\right)$ lies on a plane passing through that point, and the function approaches a definite value as we approach the point from either side of the plane, the series (4) will be summable $(C 1)$ at that point to a value half way between the limiting values of the function, provided $f(x, y, z)$ remains finite in some critical region associated with the point $\left(x_{1}, y_{1}, z_{1}\right)$. 
For the series (4) at the point $\left(x_{1}, y_{1}, z_{1}\right)$ we have

$$
\frac{S_{l m n}^{(1)}\left(x_{1}, y_{1}, z_{1}\right)}{\operatorname{lm} n}=\frac{1}{8 \operatorname{lm} n \pi^{3}} \int_{-\pi}^{\pi} \int_{-\pi}^{\pi} \int_{-\pi}^{\pi} f\left(x^{\prime}, y^{\prime}, z^{\prime}\right)
$$

$$
\frac{\sin ^{2} \frac{l\left(x^{\prime}-x_{1}\right)}{2}}{\sin ^{2} \frac{x^{\prime}-x_{1}}{2}} \frac{\sin ^{2} \frac{m\left(y^{\prime}-y_{1}\right)}{2}}{\sin ^{2} \frac{y^{\prime}-y_{1}}{2}} \frac{\sin ^{2} \frac{n\left(z^{\prime}-z_{1}\right)}{2}}{\sin ^{2} \frac{z^{\prime}-z_{1}}{2}} d x^{\prime} d y^{\prime} d z^{\prime},
$$

where $S_{l m n}^{(1)}$ is defined by equation (1). Making the change of variable

$$
x^{\prime}-x_{1}=2 \alpha, \quad y^{\prime}-y_{1}=2 \beta, \quad z^{\prime}-z_{1}=2 \gamma
$$

we have

$$
\begin{aligned}
\frac{S_{l m n}^{(1)}\left(x_{1}, y_{1}, z_{1}\right)}{l m n}= & \frac{1}{l m n \pi^{3}} \int_{-\frac{\pi+x_{1}}{2}}^{\frac{\pi-x_{1}}{2}} \int_{-\frac{\pi+y_{1}}{2}}^{\frac{\pi-y_{1}}{2}} \int_{-\frac{\pi+z_{1}}{2}}^{\frac{\pi-z_{1}}{2}} f\left(x_{1}+2 \alpha, y_{1}+2 \beta, z_{1}+2 \gamma^{\prime}\right) \\
& \cdot \frac{\sin ^{2} l \alpha}{\sin ^{2} \alpha} \frac{\sin ^{2} m \beta}{\sin ^{2} \beta} \frac{\sin ^{2} n \gamma}{\sin ^{2} \gamma} d \alpha d \beta d \gamma .
\end{aligned}
$$

In order to prove the theorem we must show that as $l, m$ and $n$ become infinite, the right-hand side of the expression (14) is equal to one-half the sum of the limiting values of the function.

Since all the points of discontinuity in the neighborhood of $\left(x_{1}, y_{1}, z_{1}\right)$ lie in a plane, we can determine a rectangular parallelepiped of dimensions $2 g$, $2 h, 2 k$ whose sides are parallel to the coördinate planes and such that the point $\left(x_{1}, y_{1}, z_{1}\right)$ lies at the center of the parallelepiped and all other points of discontinuity lie on a plane through $\left(x_{1}, y_{1}, z_{1}\right)$ either parallel to two opposite faces of the parallelepiped or passing through one of its diagonals.

If we make the transformation (13) this becomes a rectangular parallelepiped whose center lies at the point $\alpha=\beta=\gamma=0$. We may call this region $R^{\prime}$, designating the remainder of the region of integration in (14) by $R^{\prime \prime}$. We then have

$$
\frac{S_{l m n}^{(1)}\left(x_{1}, y_{1}, z_{1}\right)}{\operatorname{lmn}}=\frac{1}{\operatorname{lm} n \pi^{3}} \iiint_{R^{\prime}}+\frac{1}{\operatorname{lm} n \pi^{3}} \iiint_{R^{\prime \prime}},
$$

where the quantity under the integral signs is understood to be the same as that in equation (14). 
By virtue of Lemma 6 the second term of the right-hand side of this equation approaches zero as $l, m$ and $n$ become infinite.

The region $R^{\prime}$ is divided into two parts, $R_{1}^{\prime}$ and $R_{2}^{\prime}$, by the plane of discontinuity and hence the first term on the right-hand side of (15) may be written in the form

$$
\frac{1}{\ln n \pi^{3}}-\iiint_{R_{1}^{\prime}}+\frac{1}{\ln n \pi^{3}} \iiint_{R_{2}^{\prime}},
$$

the expression under the triple integral signs being the same as in equation (14).

If we represent by $f_{1}$ and $f_{2}$ the values which $f(x, y, z)$ approaches as we approach $\left(x_{1}, y_{1}, z_{1}\right)$ through the regions $R_{1}^{\prime}$ and $R_{2}^{\prime}$ respectively, we may write the first term of (16) in the form

$$
\begin{aligned}
& \frac{1}{\operatorname{lmn} \pi^{3}} \iiint_{R_{1}^{\prime}} \uparrow(\alpha, \beta, \gamma) \frac{\sin ^{2} l \alpha}{\sin ^{2} \alpha} \frac{\sin ^{2} m \beta}{\sin ^{2} \beta} \frac{\sin ^{2} n \gamma}{\sin ^{2} \gamma} d \alpha d \beta d \gamma \\
& +\frac{f_{1}}{\operatorname{lmn} \pi^{3}} \iiint_{R_{1}^{\prime}} \frac{\sin ^{2} l \alpha}{\sin ^{2} \alpha} \frac{\sin ^{2} m \beta}{\sin ^{2} \beta} \frac{\sin ^{2} n \gamma}{\sin ^{2} \gamma} d \alpha d \beta d \gamma,
\end{aligned}
$$

where $\varphi(\alpha, \beta, \gamma)=f\left(x_{1}+2 \alpha, y_{1}+2 \beta, z_{1}+2 \gamma\right)-f_{1}$. It then follows from Lemma 2 that the first term of this expression approaches zero as $l, m$ and $n$ become infinite. The second approaches $\frac{1}{2} f_{1}$ as $l, m$ and $n$ become infinite, by virtue of Lemma 3 if the plane of discontinuity is parallel to two opposite faces of the parallelepiped, or of Lemma 4 if it passes through a diagonal.

Similarly it may be shown that the second term of (16) approaches $\frac{1}{2} f_{2}$ as $l, m$ and $n$ become infinite and therefore (16), and hence the left-hand side of (14), approaches $\frac{1}{2}\left(f_{1}+f_{2}\right)$ as $l, m$ and $n$ become infinite and the theorem is proved.

For points on the boundaries of the region (11) except at the edges and vertices, the results are analogous to those for points in the interior and may be obtained by methods similar to those of Theorem I. These results are given in the following corollary:

COROLlaRY. If $x_{1}$ and $y_{1}$ are values of $x$ and $y$ lying in the interval $(-\pi<x<\pi,-\pi<y<\pi)$ and if $f(x, y, z)$ is integrable in the region (11) and furthermore is such that the limits

$$
\lim _{x \rightarrow x_{1}, y \rightarrow y_{1}, z \rightarrow \pi} f(x, y, z), \quad \lim _{x \rightarrow x_{1}, y \rightarrow y_{1}, z \rightarrow-\pi} f(x, y, z)
$$


exist, the Fourier development of $f(x, y, z)$ will be summable $(C 1)$ at the points $\left(x_{1}, y_{1}, \pi\right)$ and $\left(x_{1}, y_{1},-\pi\right)$ and its value will be one-half the sum of the two limits (17), provided $f(x, y, z)$ remains finite in some critical regions associated with $\left(x_{1}, y_{1}, \pi\right)$ and $\left(x_{1}, y_{1},-\pi\right)$. Analogous statements with corresponding conditions may be made for points on the other faces.

We shall next consider some of the results obtained when $\left(x_{1}, y_{1}, z_{1}\right)$ is a point of discontinuity of $f(x, y, z)$ such that all other points of discontinuity of the function in the neighborhood of that point lie on two planes through the point. When the planes are parallel to two of the coördinate planes the results are simple; this case, which is of special importance since it is analogous to the case where the points lie on the edges of the region of periodicity, is considered in the following theorem:

The0REM II. If $f(x, y, z)$ is integrable in the region (11) and $\left(x_{1}, y_{1}, z_{1}\right)$ is a point of discontinuity of $f(x, y, z)$ such that every other point of discontinuity in its neighborhood lies on one of two planes which are parallel to two coördinate planes and pass through $\left(x_{1}, y_{1}, z_{1}\right)$, and if furthermore the function approaches a definite value as we approach the point through each of the four regions into which the planes of discontinuity divide the neighborhood of that point, the series (4) will be summable $(C 1)$ at $\left(x_{1}, y_{1}, z_{1}\right)$ to a value which is one-fourth the sum of the four limiting values of the function, provided $f(x, y, z)$ remains finite in some critical region associated with the point $\left(x_{1}, y_{1}, z_{1}\right)$.

The proof of this theorem is similar to that of Theorem I. The following corollary may also be established by similar methods:

CoRollary. If $x_{1}$ is a value of $x$ lying in the interval $(-\pi<x<\pi)$ and if $f(x, y, z)$ is integrable in the region (11) and furthermore is such that the four limits

$$
\lim _{x \rightarrow x_{1} . y \rightarrow \pm \pi, z \rightarrow \pm \pi} f(x, y, z)
$$

exist, the Fourier development of $f(x, y, z)$ will be summable $(C 1)$ at each of the four points $\left(x_{1}, \pm \pi, \pm \pi\right)$ and to a value which is one-fourth the sum of the limits (18). Similar statements may be made under analogous conditions for points on the other edges.

Remark.-The conclusions of each of the first six lemmas hold equally well if instead of becoming infinite together, $l, m$ and $n$ become infinite in any other manner. Hence Theorems I and II and their accompanying corollaries hold however $l, m$ and $n$ become infinite.

In all other cases where the point of discontinuity is such that all the points of discontinuity in its neighborhood lie on two planes through the point, the value which the expression 


$$
\frac{S_{l m n}^{(1)}\left(x_{1}, y_{1}, z_{1}\right)}{\operatorname{lm} n}
$$

approaches as $l, m$ and $n$ become infinite depends on the positions of the planes and also the manner in which $l, m$ and $n$ become infinite. Before considering some of these cases it will be necessary to obtain some preliminary results.

For the purposes of our work we may divide the parallelepiped of Lemma 4 into four parts in two ways, namely by two planes each of which passes through a diagonal joining two opposite vertices of the parallelepiped or by two planes one of which passes through a diagonal and the other passes through the center and is parallel to one of the faces. We may now state the lemma:

LEMma 7. (a) If we divide the parallelepiped of Lemma 4 into four parts $R_{1}, R_{2}, R_{3}, R_{4}$ by either of the above methods, and if each of the ratios $m / n, n / l, l / m$ approaches a finite limit or becomes infinite as $l, m$ and $n$ become infinite, the limits

(20) $\lim _{l, m, n \rightarrow \infty} \frac{1}{l m n} \iiint_{R_{i}} \frac{\sin ^{2} l \alpha}{\sin ^{2} \alpha} \frac{\sin ^{2} m \beta}{\sin ^{2} \beta} \frac{\sin ^{2} n \gamma}{\sin ^{2} \gamma} d \alpha d \beta d \gamma(i=1,2,3,4)$

will exist and will each approach a limit between 0 and $\pi^{3} / 2$. Moreover the sum of two limits in which the regions of integration are adjacent is $\pi^{3} / 2$, and the limits for which the regions of integration are opposite are equal. If any of the ratios between $l, m$ and $n$ oscillates, the limits will oscillate between 0 and $\pi^{3} / 2$, their values being subject to the same restriction as above.

(b) If the intersection of the planes is parallel to the $\alpha-(\beta-$ or $\gamma-)$ axis the results are the same as above except that only the ratio $m / n(n / l$ or $l / m)$ affects the values of the limits, which are fixed if this ratio remains fixed and oscillate if it oscillates.

We shall consider, first, part $(a)$. That the value of each limit lies between 0 and $\pi^{3 / 2}$ or is equal to one of them, subject to the restriction that the sum of two limits in which the regions of integration are adjacent is equal to $\pi^{3} / 2$ : is obvious from Lemma 3 if the plane used as the dividing plane is parallel to a coördinate axis, or from Lemma 4 if it is not.

That the limits are the same for opposite regions of integration may be seen by making suitable changes of variable of the type $\alpha=-\alpha^{\prime}$.

Each of the limits (20) depends on a limit of the type

$$
\lim _{l, m, n \rightarrow \infty} \frac{1}{l m n} \int_{0}^{g} \int_{0}^{p \alpha} \int_{0}^{q \alpha+r \beta} \frac{\sin ^{2} l \alpha}{\sin ^{2} \alpha} \frac{\sin ^{2} m \beta}{\sin ^{2} \beta} \frac{\sin ^{2} n \gamma}{\sin ^{2} \gamma} d \alpha d \beta d \gamma
$$


which, if we apply Lemma 5 and make the change of variable

$$
l \alpha=\alpha^{\prime}, \quad m \beta=\beta^{\prime}, \quad n \gamma=\gamma^{\prime},
$$

reduces to the form

$$
\lim _{l, m, n \rightarrow \infty} \int_{0}^{l g} \int_{0}^{\frac{m}{l} p \alpha} \int_{0}^{\frac{n}{l} q \alpha+\frac{n}{m} r \beta} \frac{\sin ^{2} \alpha}{\alpha^{2}} \frac{\sin ^{2} \beta}{\beta^{2}} \frac{\sin ^{2} \gamma}{\gamma^{2}} d \alpha d \beta d \gamma .
$$

This limit depends on the values of the ratios between $l, m$ und $n$ and hence when any of these ratios oscillates the value of the limit oscillates.

To prove the lemma for case (b) we may assume that the intersection of the planes is parallel to the $\alpha$-axis. Each of the limits (20) depends on a limit of the type

$$
\lim _{l, m, n \rightarrow \infty} \frac{1}{l m n} \int_{0}^{g} \int_{0}^{h} \int_{0}^{k \beta} \frac{\sin ^{2} l \alpha}{\sin ^{2} \alpha} \frac{\sin ^{2} m \beta}{\sin ^{2} \beta} \frac{\sin ^{2} n \gamma}{\sin ^{2} \gamma} d \alpha d \beta d \gamma
$$

which reduces to the form

$$
\int_{0}^{\infty} \frac{\sin 2 \alpha}{\alpha^{2}}\left\{\int_{0}^{\infty} \frac{\sin ^{2} \beta}{\beta^{2}}\left(\int_{0}^{r \beta} \frac{\sin ^{2} \gamma}{\gamma^{2}} d \gamma\right) d \beta\right\} d \alpha
$$

by Lemma 5 and the change of variable (21) if we set

$$
r=\lim _{m, n \rightarrow \infty} \frac{n}{m} k .
$$

The proof then follows as in part $(a)$.

In this case we may obtain an exact value for the limits (20) since they depend on triple integrals of the type of (22), which we may evaluate without great difficulty. The double integral in brackets in (22) has been evaluated by C. N. Moore*, and applying his results we have for the value of (22)

$$
\begin{gathered}
\frac{\pi^{3}}{8}+\frac{\pi}{2}\left\{\frac{(r+1) \log (r+1)-2 r \log r+(r-1) \log (r-1)}{4}\right. \\
\left.+\frac{(r-1) \log (r-1)-(r+1) \log (r+1)}{4 r}-\left(\frac{1}{r}+\frac{1}{3^{2} r^{3}}+\frac{1}{5^{2} r^{5}}+\cdots\right)\right\}(r>1), \\
\frac{\pi^{3}}{16} \quad(r=1), \\
\frac{\pi}{2}\left\{\frac{(1+r) \log (1+r)-2 r \log r-(1-r) \log (1-r)}{4}\right. \\
\left.-\frac{(1-r) \log (1-r)+(1+r) \log (1+r)}{4 r}+\left(r+\frac{1}{3^{2}} r^{3}+\frac{1}{5^{2}} r^{5}+\cdots\right)\right\}(0<r<1) .
\end{gathered}
$$

${ }^{*}$ Loc. cit. p. 564. 
We may now consider the summability of $f(x, y, z)$ at a point of discontinuity such that all other points of discontinuity in its neighborhood lie on two planes both of which are not parallel to a coördinate plane. The proofs may easily be carried out by methods similar to those of Theorem I except that Lemma 7 is used instead of Lemmas 3 and 4.

Let $f(x, y, z)$ be a function that is integrable throughout the region (11) and $\left(x_{1}, y_{1}, z_{1}\right)$ a point of discontinuity of $f(x, y, z)$ such that all the discontinuities in its neighborhood lie on two planes which pass through that point and whose intersection is not parallel to a coördinate axis, and furthermore let $f(x, y, z)$ remain finite in some critical region associated with $\left(x_{1}, y_{1}, z_{1}\right)$ and approach a definite value as we approach $(x, y, z)$ through each of the regions into which the planes of discontinuity divide the neighborhood of that point. Then if $l, m$ and $n$ become infinite in such a manner that any of the ratios $n / m, l / n, m / l$ oscillates, the value of (19) will oscillate between $\frac{1}{2}\left(f_{1}+f_{3}\right)$ and $\frac{1}{2}\left(f_{2}+f_{4}\right)$, where $f_{1}, f_{2}, f_{3}, f_{4}$ are the values which the function approaches, assigned in rotation. If the ratios become infinite or remain finite the expression (19) will approach a finite limit between $\frac{1}{2}\left(f_{1}+f_{3}\right)$ and $\frac{1}{2}\left(f_{2}+f_{4}\right)$ or equal to one of them.

If $f(x, y, z)$ and $\left(x_{1}, y_{1}, z_{1}\right)$ satisfy the conditions of the preceding paragraph except that the intersection of the planes of discontinuity is parallel to one of the coördinate axes, the only ratio which affects the limit of the expression (19) is $n / m, l / n, m / l$, according as the intersection of the planes is parallel to the $x$-, $y$ - or $z$-axis. If this ratio oscillates the value of the limit (19) will oscillate between $\frac{1}{2}\left(f_{1}+f_{3}\right)$ and $\frac{1}{2}\left(f_{2}+f_{4}\right)$, and if it approaches a finite limit or becomes infinite (19) will approach a limit between these values or equal to one of them. The exact value may be obtained by determining the values of the limits (20) from the expressions for the integral (22).

In particular we may derive the following theorem:

THEOREM III. Let $f(x, y, z)$ be a function which is integrable in the region (11) and $\left(x_{1}, y_{1}, z_{1}\right)$ a point of discontinuity of $f(x, y, z)$ such that every other point of discontinuity in its neighborhood lies on two mutually perpendicular planes which pass through $\left(x_{1}, y_{1}, z_{1}\right)$ and whose intersection is parallel to one of the coördinate axes. Then if $f(x, y, z)$ remains finite in some critical region associated with $\left(x_{1}, y_{1}, z_{1}\right)$ and approaches a definite value as we approach that point through each of the four regions into which the planes of discontinuity divide its neighborhood, the series (4) will be summable $\left(C^{\prime} 1\right)$ at $\left(x_{1}, y_{1}, z_{1}\right)$ to one-fourth the sum of the four limiting values of the function, provided the ratio $n / m, l / n$, or $m / l$, according as the intersection of the planes of discontinuity is parallel to the $x-, y$-, or $z$-axis, approaches unity as $l, m$ and $n$ become infinite. 
Results similar to those obtained in the above discussion hold where the discontinuities are distributed on a broken plane instead of on two intersecting planes.

We may also carry through discussions of the cases where the discontinuities lie on three or more planes intersecting at a point of discontinuity, but as these results are in general complicated we shall confine ourselves to stating one particular case from which we may deduce the behavior of the series at the vertices of the region (11). The results are contained in the following theorem and its corollary, the proofs being carried out by a method similar to that used in Theorem I:

THEOREM IV. If $f(x, y, z)$ is integrable in the region (11) and $\left(x_{1}, y_{1}, z_{1}\right)$ is a point of discontinuity of $f(x, y, z)$ such that every other point of discontinuity in its neighborhood lies on three planes through that point and parallel respectively to the coördinate planes, and if moreover $f(x, y, z)$ approaches a definite value as we approach $\left(x_{1}, y_{1}, z_{1}\right)$ through each of the eight regions into which the planes divide the neighborhood of that point, the series (4) will be summable $(C 1)$ at the point $\left(x_{1}, y_{1}, z_{1}\right)$ and to a value which is one-eighth the sum of the eight limiting values of the function, provided $f(x, y, z)$ remains finite in some critical region associatcd with the point $\left(x_{1}, y_{1}, z_{1}\right)$.

COROLLARY. If $f(x, y, z)$ is integrable in the region (11) and if the eight limits

$$
\lim _{x \rightarrow \pm \pi, y \rightarrow \pm \pi, z \rightarrow \pm \pi} f(x, y, z)
$$

exist, the series (4) will be summable $(C 1)$ at the eight vertices of the region (11) and its value will be one-eighth the sum of the eight limits (23), provided the function remains finite in some critical region of each of the eight vertices.

\section{Discontinuities Which LIE ALONG CURVEd SURFaces}

We shall now consider the behavior of the series at a point of discontinuity such that all other points of discontinuity in its neighborhood lie on a curved surface through the point and such that the function $f(x, y, z)$ approaches a definite value as we approach the point of discontinuity from either side of the surface. This surface is assumed to have a tangent plane at the point of discontinuity under consideration and to be of such nature that any plane through the point intersects the surface in a finite number of curves or not at all.

Before proving our main theorem we shall have to prove several lemmas.

Lemma 8. The integral

$$
\int_{0}^{\infty} \int_{0}^{\infty} \int_{0}^{\dot{\alpha} \alpha+\mu \beta} \frac{\sin ^{2} \alpha}{\alpha^{2}} \frac{\sin ^{2} \beta}{\beta^{2}} \frac{\sin ^{2} \gamma}{\gamma^{2}} d \alpha d \beta d \gamma
$$


converges for all values of $\lambda$ and $\mu$ and represents a function of $\lambda$ and $\mu$, $\psi(\lambda, \mu)$, which is continuous for all values of $\lambda$ and $\mu$ and approaches $\pi^{3} / 8$ as a limit as $\lambda$ and $\mu$ become infinite in the same or opposite directions.

We shall consider first the case where $\lambda$ and $\mu$ are both positive. If we let

$$
\varphi(\lambda, \mu, \alpha, \beta)=\int_{0}^{\lambda \alpha+\mu \beta} \frac{\sin ^{2} \gamma}{\gamma^{2}} d \gamma
$$

we may write the integral (24) in the form

$$
\int_{0}^{\infty} \int_{0}^{\infty} \varphi(\lambda, \mu, \alpha, \beta) \frac{\sin ^{2} \alpha}{\alpha^{2}} \frac{\sin ^{2} \beta}{\beta^{2}} d \alpha d \beta .
$$

It may readily be seen that $\varphi(\lambda, \mu, \alpha, \beta)$ is a continuous function of $\lambda, \mu, \alpha, \beta$ for all values of these arguments and that it remains finite for all such values. Therefore the integrand of (25) is a continuous function and as the double integral (25) is uniformly convergent, $\psi(\lambda, \mu)$, the function represented by (25), defines a function of $\lambda$ and $\mu$ which is continuous for all values of $\lambda$ and $\mu$.

If $\lambda$ and $\mu$ are of opposite sign, say $\lambda$ is negative, we may make the substitution $\alpha=-\alpha^{\prime}$ and the proof follows in the same manner as before. If both $\lambda$ and $\mu$ are negative, we set $\alpha=-\alpha^{\prime}, \beta=-\beta^{\prime}$. and the proof follows similarly.

Lемма 9. The integral

$$
\int_{0}^{\infty} \int_{0}^{-\frac{\lambda}{\mu} \alpha} \int_{0}^{\lambda \alpha+\mu \beta} \frac{\sin ^{2} \alpha}{\alpha^{z}} \frac{\sin ^{2} \beta}{\beta^{2}} \frac{\sin ^{2} \gamma}{\gamma^{2}} d \alpha d \beta d \gamma
$$

where $\mu$ remains fixed, converges for all values of $\lambda$ and represents a finction of $\lambda, \Omega(\lambda)$, which is continuous for all values of $\lambda$ and remains finite as $\lambda$ becomes positively or negatively infinite.

Defining

$$
\int_{0}^{-\frac{\lambda}{\mu} \alpha} \int_{0}^{\lambda \alpha+\mu \beta} \frac{\sin ^{2} \beta}{\beta^{2}} \frac{\sin ^{2} \gamma}{\gamma^{2}} d \beta d \gamma
$$

by $\chi(\lambda, \alpha)$, we may write the integral (26) in the form

$$
\int_{0}^{\infty} \chi(\lambda, \alpha) \frac{\sin ^{2} \alpha}{\alpha^{2}} d \alpha
$$


It is obvious that $\chi(\lambda, \alpha)$ is a continuous function of $\lambda$. and $\alpha$ for all values of these arguments and that it remains finite for all such values. Hence the integrand of (27) is a continuous function of $\lambda$ and $\alpha$ for all values of these arguments and since the integral is uniformly convergent for all values of $\lambda$, it renresents a function, $\Omega(\lambda)$, which is continuous for all values of $\lambda$.

As $\lambda$ becomes infinite $\chi(\lambda, \alpha)$ approaches the limit $\pi^{2} / 4$, provided $\alpha \neq 0$, and hence $\boldsymbol{\Omega}(\lambda)$ approaches $\pi^{3} / 8$ as a limit and therefore remains finite for all positive values of $\lambda$.

For the case where $\lambda$ is negative, the proof may be carried out by making the change of variable $\alpha=-\alpha^{\prime}$.

Lemma 10. If $\gamma=G(\alpha, \beta)$ represents a curved surface whose tangent plane at the origin is $\gamma=\lambda \alpha+\mu \beta$ and whose intersection with the $\alpha, \beta$ plane is $\beta=g(\alpha)$, and if $\gamma=G(\alpha, \beta)$ is intersected in only a finite number of curves in the neighborhood of the origin by any plane passing through that point. tho limits

$$
\begin{array}{r}
\left.-\frac{1}{l m n} \int_{0}^{h} \int_{0}^{k} \int_{0}^{\lambda \alpha+\mu \beta} \frac{\sin ^{2} \alpha}{\alpha^{2}} \frac{\sin ^{2} \beta}{\beta^{2}} \frac{\sin ^{2} \gamma}{\gamma^{2}} d \alpha d \beta d \gamma\right], \\
\lim _{l, m, n \rightarrow \infty}\left[\frac{1}{l m n} \int_{0}^{h} \int_{0}^{g(\alpha)} \int_{0}^{G(\alpha, \beta)} \frac{\sin ^{2} \alpha}{\alpha^{2}} \frac{\sin ^{2} \beta}{\beta^{2}} \frac{\sin ^{2} \gamma}{\gamma^{2}} d \alpha d \beta d \gamma\right. \\
\left.-\frac{1}{l m n} \int_{0}^{h} \int_{0}^{-\frac{\lambda}{\mu} \alpha} \int_{0}^{\lambda \alpha+\mu \beta} \frac{\sin ^{2} \alpha}{\alpha^{2}} \frac{\sin ^{2} \beta}{\beta^{2}} \frac{\sin ^{2} \gamma}{\gamma^{2}} d \alpha d \beta d \gamma\right],
\end{array}
$$$$
\lim _{l, m, n \rightarrow \infty}\left[\frac{1}{l m n} \int_{0}^{n} \int_{0}^{k} \int_{0}^{G(\alpha, \beta)} \frac{\sin ^{2} \alpha}{\alpha^{2}} \frac{\sin ^{2} \beta}{\beta^{2}} \frac{\sin ^{2} \gamma}{\gamma^{2}} d \alpha d \beta d \gamma\right.
$$

where $h$ and $k$ are constants between 0 and $\pi$, will exist and be equal to zero provided that $\lambda$ and $\mu$ are not zero in (28) and that $\mu$ is not zero in (29).*

We shall carry out the proof where $\lambda$ and $\mu$ are both positive, the other cases following by a change of variable as in Lemmas 8 and 9 .

Consider first the expression (28), writing the quantity in brackets in the form

* If the equation of the surface or tangent plane is such that it can not be put in the form used in the statement of the lemma, we may use $\alpha$ or $\beta$ as the left-hand side of the equations, modifying the remainder of the statement and the proof accordingly. 


$$
\begin{array}{r}
\quad \frac{1}{\operatorname{lm} n}\left\{\int_{0}^{\rho} \int_{0}^{\partial q} \int_{0}^{\sigma(\alpha, \beta)}-\int_{0}^{\rho p} \int_{0}^{\partial q} \int_{0}^{\partial \alpha \alpha+\mu \beta}\right\} \\
+\frac{1}{\operatorname{lm} n}\left\{\int_{0}^{h} \int_{0}^{k} \int_{0}^{\circ G(\alpha, \beta)}-\int_{0}^{\rho p} \int_{0}^{q} \int_{0}^{\partial G(\alpha, \beta)}\right\} \\
+\frac{1}{\ln n}\left\{\int_{0}^{p} \int_{0}^{\rho q} \int_{0}^{\partial \lambda \alpha+\mu \beta}-\int_{0}^{h} \int_{0}^{k} \int_{0}^{\partial \alpha \alpha+\mu \beta}\right\},
\end{array}
$$

where $0<p<h, 0<q<k$, the expression under the triple integral signs being the same as that in (28). It is obvious that the second and third terms of (30) approach zero as $l, m$ and $n$ become infinite. Then if we can show that $p$ and $q$ may be so chosen that for sufficiently large values of $l, m$ and $n$ the first term of (30) may be made as small as we please in absolute value, the lemma will have been demonstrated for the expression (28).

Assume for the sake of definiteness that the portion of the surface above the $\alpha, \beta$ plane lies above the tangent plane until possibly it intersects it. We may choose a $\lambda^{\prime}$ and $\mu^{\prime}$ greater respectively than $\lambda$ and $\mu$, such that $\lambda^{\prime} / \mu^{\prime}=\lambda / \mu$ and such that the plane $\gamma=\lambda^{\prime} \alpha+\mu^{\prime} \beta$ intersects the surface in a curve whose projection on the $\alpha, \beta$ plane lies entirely within the projection of any curve in which the tangent may cut the surface in the octant $0-X Y Z$. If we draw a rectangle two of whose sides lie along the $\alpha$ and $\beta$ axes, within the area included between these axes and the projection of the intersection of $\gamma=\lambda^{\prime} \alpha+\mu^{\prime} \beta$ and $\gamma=G(\alpha, \beta)$ in this octant, the vertex opposite the origin being $(p, q, 0)$, we have

$$
\frac{1}{\operatorname{lm} n} \int_{0}^{\rho p} \int_{0}^{q} \int_{0}^{\lambda^{\prime} \alpha+\mu^{\prime} \beta}>\frac{1}{\operatorname{lm} n} \int_{0}^{p} \int_{0}^{q} \int_{0}^{G(\alpha, \beta)}>\frac{1}{\operatorname{lm} n} \int_{0}^{p} \int_{0}^{q} \int_{0}^{\lambda \alpha+\mu \beta}
$$

the expression under the integral signs being the same as that in (28). Making the transformation (21) we see that, $p$ and $q$ being fixed, we can choose $l$ and $m$ so large that the first and third members of the inequality (31) become as near $\psi\left(n \lambda^{\prime} / l, n \mu^{\prime} / m\right)$ and $\psi(n \lambda / l, n \mu / m)$ respectively as we wish, this function being defined as in Lemma 8 . But since by that lemma this function is continuous, it follows that for $\lambda^{\prime}$ and $\mu^{\prime}$ sufficiently near to $\lambda$ and $\mu$ respectively, $\psi\left(n \lambda^{\prime} / l, n \mu^{\prime} / m\right)$ and $\psi(n \lambda / l, n \mu / m)$ differ for all values of $l, m$ and $n$ by as small a quantity as we please.*

* The restriction $\lambda \neq 0, \mu \neq 0$ is of course essential for this conclusion. When these conditions do not hold, we must limit the freedom of variation of $l, m$ and $n$ as is pointed out in Lemma 11. 
Hence from (31) $p$ and $q$ may be so chosen that for $l, m$ and $n$ large enough the first term of (30) is as small in absolute value as we please, and the lemma is proved for the expression (28).

To prove it for (29) we write the quantity in brackets in that expression in the form

$$
\begin{aligned}
& \quad \frac{1}{\operatorname{lm} n}\left\{\int_{0}^{r} \int_{0}^{g(\alpha)} \int_{0}^{. G(\alpha, \beta)}-\int_{0}^{r} \int_{0}^{-\frac{\lambda}{\mu} \alpha} \int_{0}^{\lambda \alpha+\mu \beta}\right\} \\
& +\frac{1}{\operatorname{lm} n}\left\{\int_{0}^{h} \int_{0}^{g(\alpha)} \int_{0}^{G(\alpha, \beta)}-\int_{0}^{r} \int_{0}^{g(\alpha)} \int_{0}^{G(\alpha, \beta)}\right\} \\
& +\frac{1}{\ln n}\left\{\int_{0}^{r} \int_{0}^{-\frac{\lambda}{\mu} \alpha} \int_{0}^{\lambda \alpha+\mu \beta}-\int_{0}^{h} \int_{0}^{-\frac{\lambda}{\mu} \alpha} \int_{0}^{\lambda \alpha+\mu \beta}\right\},
\end{aligned}
$$

where $0<r<h$, the expression under the triple integral signs being the same as that in (29).

Here as in (30) the second and third terms approach zero as $l, m$ and $n$ become infinite. Then we have only to show that $r$ may be so chosen that for sufficiently large values of $l, m$ and $n$ the first term of (32) may be made as small in absolute value as we please, and the lemma will have been proved for the expression (29).

We shall again use the surface which we used in the proof for the expression (28), considering that part which lies in the octant $0-X Y^{\prime} Z$. We may then choose a $\lambda^{\prime}$ greater than $\lambda$ and such that the plane $\gamma=\lambda^{\prime} \alpha+\mu \beta$ intersects the surface in a curve whose projection on the $\alpha, \beta$ plane in the octant $0-X Y^{\prime} Z$ lies entirely within the projection of any curve in which the tangent plane may cut the surface in that octant. Then if $r$ is the $\alpha$ coördinate of the first point to the right of the origin in which the tangent plane intersects the surface, we have

$$
\begin{aligned}
\frac{1}{\operatorname{lm} n} \int_{0}^{r} \int_{-\frac{\lambda^{\prime}}{\mu} \alpha}^{0} \int_{0}^{\lambda^{\prime} \alpha+\mu \beta} & >\frac{1}{\operatorname{lm} n} \int_{0}^{r} \int_{g(\alpha)}^{0} \int_{0}^{G(\alpha, \beta)} \\
& >\frac{1}{\operatorname{lm} n} \int_{0}^{r} \int_{-\frac{\lambda}{\mu} \alpha}^{0} \int_{0}^{\lambda \alpha+\mu \beta}
\end{aligned}
$$

where the expression under the integral signs is the same as before. 
Making the transformation (21) we proceed by a method analogous to that used in the proof for the expression (28), using Lemma 9 instead of Lemma 8, and the lemma is proved for the expression (29).

Lемма 11. If all the conditions of Lemma 10 are satisfied by $\gamma=G(\alpha, \beta)$ except that the tangent to the surface passes through the $\alpha$-or $\beta$-axis or coincides with the $\alpha, \beta$ plane we have the following results:

(a) if the tangent plane passes through the $\alpha$-axis $(\lambda=0)$, the limit (28) exists and is equal to zero provided $n$ and $l$ become infinite in such a manner that

$$
n / l<K
$$

where $K$ is a positive constant. Similarly if the tangent passes through the $\beta$-axis $(\mu=0)$, the limit (28) exists and is equal to zero provided that $n$ and $m$ become infinite in such a manncr that

$$
n / m<K
$$

(b) if the tangent plane passes through the $\alpha$-axis the limit

$$
\lim _{l, m, n \rightarrow \infty} \frac{1}{\operatorname{lm} n} \int_{0}^{h} \int_{g(\alpha)}^{\infty} \int_{0}^{G(\alpha, \beta)} \frac{\sin ^{2} \alpha}{\alpha^{2}} \frac{\sin ^{2} \beta}{\beta^{2}} \frac{\sin ^{2} \gamma}{\gamma^{2}} d \alpha d \beta d \gamma
$$

will exist and be equal to zero provided $l, m$ and $n$ become infinite in such a way that the ratio between any two of them remains finite;

(c) if the tangent plane coincides with the $\alpha, \beta$ plane the limit

$$
\lim _{l, m, n \rightarrow \infty} \frac{1}{l m n} \int_{0}^{h} \int_{0}^{k} \int_{0}^{G(\alpha, \beta)} \frac{\sin ^{2} \alpha}{\alpha^{2}} \frac{\sin ^{2} \beta}{\beta^{2}} \frac{\sin ^{2} \gamma}{\gamma^{2}} d \alpha d \beta d \gamma
$$

will be exist and be equal to zero provided $l, m$ and $n$ become infinite as provided in (b).

The proofs involved in this lemma may be obtained by making slight changes in Lemma 10. For example, in the first part of $(a)$ where $\lambda=0$, we cannot say that if $\lambda^{\prime}$ is taken near enough to $\lambda, \psi\left(n \lambda^{\prime} / l, n \mu^{\prime} / m\right)$ and $\psi(n \lambda / l, n \mu / m)$ differ by as small a quantity as we please for all values of $l, m$ and $n$. The statement is true for only such values of $l$ and $n$ as satisfy the inequality (34). The remaining proofs may be carried out by making similar changes in Lemma 10.

Before proceeding with the next theorem we shall define a term which we wish to use in the statement of the theorem. If the quotient in (3), corresponding to a triple series $\sum a_{l m n}$, approaches a limit as $l, m$ and $n$ 
become infinite provided the ratios between $l, m$ and $n$ remain less in absolute value than a positive constant, we say that the triple series is restrictedly summable $(C r)$ and that its limit is the value approached. If only one ratio needs to be restricted we say that the series is restrictedly summable with respect to the quantities involved in that ratio.

Theorem V. (a) Let $f(x, y, z)$ be a function which is integrable throughout the region (11) and $\left(x_{1}, y_{1}, z_{1}\right)$ a point of discontinuity of $f(x, y, z)$ such that every other point of discontinuity in its neighborhood lies on a surface satisfying the following conditions: (1) the surface has a tangent plane at the point $\left(x_{1}, y_{1}, z_{1}\right)$ which is not parallel to any of the coördinate axes; (2) no plane through the point $\left(x_{1}, y_{1}, z_{1}\right)$ intersects the surface in an infinite number of curves in the neighborhood of that point; and (3) the function $f(x, y, z)$ approaches a definite value as we approach $\left(x_{1}, y_{1}, z_{1}\right)$ from either side of the surface. Then the series (4) will be summable $(C 1)$ at the point $\left(x_{1}, y_{1}, z_{1}\right)$ to a value half way between the limiting values of the function at that point, provided $f(x, y, z)$ remains finite in some critical region associated with $\left(x_{1}, y_{1}, z_{1}\right)$.

(b) If all the other conditions are fulfilled, but the tangent plane to the surface at $\left(x_{1}, y_{1}, z_{1}\right)$ is parallel to a coördinate axis or plane, the series will be restrictedly summable to one-half the sum of the limiting values of the function at $\left(x_{1}, y_{1}, z_{1}\right)$. If, however, the plane tangent to the surface at $(x, y, z)$ is parallel to only one of the coördinate axes and remains tangent to the surface in a line parallel to that axis, only one ratio needs to be restricted, the ratio being $n / m, l / n, m / l$, according as the line of tangency is parallel to the $x$-, $y$-, or $z$-axis.

We shall consider first the case $(a)$. We may choose a rectangular parallelepiped $R$ with $\left(x_{1}, y_{1}, z_{1}\right)$ as its center, such that the tangent plane passes through one diagonal and all points of discontinuity in the parallelepiped lie on the surface of discontinuity, and small enough so that the surface of discontinuity does not intersect the plane tangent to the surface within the parallelepiped, except perhaps at the point of tangency.

The Fourier series corresponding to $f(x, y, z)$ may then be written

$$
\begin{aligned}
& \frac{S_{l m n}^{(1)}\left(x_{1}, y_{1}, z_{1}\right)}{\operatorname{lm} n}=\frac{1}{\operatorname{lm} n \pi^{3}} \iiint_{R^{\prime}} f\left(x_{1}+2 \alpha, y_{1}+2 \beta, z_{1}+2 \gamma\right) \\
& \cdot \frac{\sin ^{2} l \alpha}{\sin ^{2} \alpha} \frac{\sin ^{2} m \beta}{\sin ^{2} \beta} \frac{\sin ^{2} n \gamma}{\sin ^{2} \gamma} d \alpha d \beta d \gamma \\
& +\frac{1}{\operatorname{lm} n \pi^{3}} \iiint_{R^{\prime \prime}} f\left(x_{1}+2 \alpha, y_{1}+2 \beta, z_{1}+2 \gamma\right) \\
& \text {. } \frac{\sin ^{2} l \alpha}{\sin ^{2} \alpha} \frac{\sin ^{2} m \beta}{\sin ^{2} \beta} \frac{\sin ^{2} n \gamma}{\sin ^{2} \gamma} d \alpha d \beta d \gamma,
\end{aligned}
$$


where $R^{\prime}$ represents the rectangular parallelepiped into which $R$ is transformed by the change of variable (13) and $R^{\prime \prime}$ is the remainder of the region of integration of the integral (14). The second term of (35) vanishes as $l, m$ and $n$ become infinite, by Lemma 1 . Then in order to prove our theorem we have only to show that as $l, m$ and $n$ become infinite the first term approaches a value which is one-half the sum of the limiting values of $f(x, y, z)$ at $\left(x_{1}, y_{1}, z_{1}\right)$.

Let the surface of discontinuity divide the parallelepiped into two parts, $R_{1}^{\prime}$ and $R_{2}^{\prime}$ and let $f_{1}$ and $f_{2}$ be the limiting values of the function as we approach the point of discontinuity through $R_{1}^{\prime}$ and $R_{2}^{\prime}$ respectively. Then the first term of (35) may be written

$$
\frac{1}{\operatorname{lm} n \pi^{3}} \iiint_{R_{1}^{\prime}}+\frac{1}{\operatorname{lm} n \pi^{3}} \iiint_{R_{2}^{\prime}}
$$

where the quantity under the triple integral signs is the same as in (35). We shall show that the first term of (36) approaches $\frac{1}{2} f_{1}$ as a limit as $l, m$ and $n$ become infinite.

The tangent plane to the surface at $\left(x_{1}, y_{1}, z_{1}\right)$ divides the region $R_{1}^{\prime}$ into two parts; let $D_{1}$ be that one which lies on the same side of the tangent plane as $R_{1}^{\prime}$ does of the surface of discontinuity. Then by Lemma 10

$$
\begin{array}{r}
\frac{1}{\operatorname{lm} n} \iiint_{D_{1}} \frac{\sin ^{2} l \alpha}{\alpha^{2}} \frac{\sin ^{2} m \beta}{\beta^{2}} \frac{\sin ^{2} n \gamma}{\gamma^{2}} d \alpha d \beta d \gamma \\
-\frac{1}{l m n} \iiint_{R_{1}^{\prime}} \frac{\sin ^{2} l \alpha}{\alpha^{2}} \frac{\sin ^{2} m \beta}{\beta^{2}} \frac{\sin ^{2} n \gamma}{\gamma^{2}} d \alpha d \beta d \gamma
\end{array}
$$

approaches zero as $l, m$ and $n$ become infinite, and hence by Lemma 5

$$
\begin{array}{r}
\frac{1}{\operatorname{lm} n} \iiint_{D_{1}} \frac{\sin ^{2} l \alpha}{\sin ^{2} \alpha} \frac{\sin ^{2} m \beta}{\sin ^{2} \beta} \frac{\sin ^{2} n \gamma}{\sin ^{2} \gamma} d \alpha d, \beta d \gamma \\
-\frac{1}{l m n} \iiint_{R_{1}^{\prime}} \frac{\sin ^{2} l \alpha}{\sin ^{2} \alpha} \frac{\sin ^{2} m \beta}{\sin ^{2} \beta} \frac{\sin ^{2} n \gamma}{\sin ^{2} \gamma} d \alpha d \beta d \gamma
\end{array}
$$

approaches zero as $l, m$ and $n$ become infinite. But by Lemma 4 the first term of (37) approaches $\pi^{3} / 2$ and hence the second term must also approach $\pi^{3} / 2$ as $l, m$ and $n$ become infinite. Therefore the first term of (36) approaches $\frac{1}{2} f_{1}$ as $l, m$ and $n$ become infinite. Similarly it may be shown that the second 
term of (36) approaches the value $\frac{1}{2} f_{2}$ and therefore the whole expression (36) and hence the right-hand side of (35) approaches $\frac{1}{8}\left(f_{1}+f_{2}\right)$ as $l, m$ and $n$ become infinite, and part $(a)$ of the theorem is proved. Part $(b)$ may be proved similarly by using Lemma 10 instead of Lemma 9.

We wish now to show that restricted summability is all that may be established for part $(b)$. Since the proofs are analogous for the cases where the tangent plane is parallel to a coördinate axis or to a corrdinate plane, we shall give the proof only for the latter, considering the development of a function that is continuous throughout the region (11) except on a curved surface lying above the $x, y$ plane and tangent to it at the origin, and that approaches $f_{1}$ as we approach the surface of discontinuity from above the surface and $f_{2}$ as we approach it from below.

From Moore's generalization of Fejér's theorem,

$$
\begin{aligned}
f\left(x^{\prime}, 0,0\right)=\sum_{m=1, n=1}^{\infty, \infty} & \frac{1}{2^{E(1 / m)+} \overline{E(1 / n)} \pi^{2}} \int_{-\pi}^{\pi} \int_{-\pi}^{\pi} f\left(x^{\prime}, y^{\prime}, z^{\prime}\right) \\
& \cdot \cos \left[(m-1) y^{\prime}\right] \cos \left[(n-1) z^{\prime}\right] d x^{\prime} d y^{\prime} d z^{\prime} \quad\left(x^{\prime} \neq 0\right) .
\end{aligned}
$$

The series on the right may be integrated term by term if we multiply it by a continuous function of $x$, and therefore

$$
\begin{aligned}
& \frac{1}{2^{E(1 / l)} \pi} \int_{-\pi}^{\pi} f\left(x^{\prime}, 0,0\right) \cos \left[(l-1) x^{\prime}\right] d x^{\prime} \\
& =\sum_{m=1, n=1}^{\infty, \infty} \frac{1}{2^{E(1 / l)+E(1 / m)+E(1 / n)} \pi^{3}} \int_{-\pi}^{\pi} \int_{-\pi}^{\pi} \int_{-\pi}^{\pi} f\left(x^{\prime}, y^{\prime}, z^{\prime}\right) \\
& \quad \cos \left[(l-1) x^{\prime}\right] \cos \left[(m-1) y^{\prime}\right] \cos \left[(n-1) z^{\prime}\right] d x^{\prime} d y^{\prime} d z^{\prime},
\end{aligned}
$$

and by the generalization of Fejér's theorem,

$$
\begin{aligned}
& \sum_{l=1}^{\infty}\left\{\sum_{m=1, n=1}^{\infty} \frac{1}{2^{E(1 / l)+E(1 / m)+E(1 / n)} \pi^{3}} \int_{-\pi}^{\pi} \int_{-\pi}^{\pi} \int_{-\pi}^{\pi} f\left(x^{\prime}, y^{\prime}, z^{\prime}\right)\right. \\
& \left.\cdot \cos \left[(l-1) x^{\prime}\right] \cos \left[(m-1) y^{\prime}\right] \cos \left[(n-1) z^{\prime}\right] d x^{\prime} d y^{\prime} d z^{\prime}\right\} \\
& =\sum_{l=1}^{\infty} \frac{1}{2^{E(1 / l)} \pi} \int_{-\pi}^{\pi} f\left(x^{\prime}, 0,0\right) \cos \left[(l-1) x^{\prime}\right] d x^{\prime}=f_{2}
\end{aligned}
$$


and the expression $(1 / l m n)\left[S_{l m n}^{(1)}(0,0,0)\right]$ approaches $f_{2}$ if we let $m$ and $n$ become infinite holding $l$ fixed, and then let $l$ become infinite. If we let $l$ and $n$ become infinite holding $m$ fixed and then let $m$ become infinite the series is again summable to $f_{\mathbf{z}}$ at the origin, but if we let $l$ and $m$ become infinite and then $n$, the value to which the series is summable at the origin is $\frac{1}{2}\left(f_{1}+f_{2}\right)$ and hence the triple series cannot be summable in the ordinary sense. As pointed out above, it may be shown in analogous fashion that restricted summability is all that can be proved when the tangent plane is parallel to one coordinate axis.

By methods similar to those of Theorem $\mathrm{V}$ and its preliminary lemmas we may obtain the value to which the function is summable at points of discontinuity such that all other points of discontinuity in their neighborhood are on two or more curved surfaces through the point, or lie at the intersection of two or more such surfaces. The results in these cases are analogous to those of Theorems II, III and IV, except that when the tangent to one or more of the curved surfaces is parallel to a coordinate axis we have restricted instead of ordinary summability.

University of Cincinnati, Cincinnati, 0hio. 